ALINE ROCHA GUERIN ${ }^{1}$

Reginaldo Guedes Coelho Lopes²

Daniella de Batista Depes ${ }^{3}$

JoÃo AlfREDO MARTINS ${ }^{4}$

\title{
Achados histeroscópicos na cavidade endometrial após ablação endometrial
}

\author{
Hysteroscopic appearance of the endometrial cavity after \\ endometrial ablation
}

Artigo Original

\section{Palavras-chave \\ Histeroscopia \\ Técnicas de ablação endometrial \\ Aderências focais \\ Hematometra \\ Keywords \\ Hysteroscopy \\ Endometrial ablation techniques \\ Focal adhesions \\ Hematometra}

\section{Resumo}

OBJETIVO: Verificar o aspecto da cavidade uterina após a ablação endometrial histeroscópica, a prevalência de sinéquias após o procedimento e, com isso, avaliar a importância da histeroscopia realizada no pós-operatório dessas pacientes. MÉTODOS: Foram avaliados, retrospectivamente, os laudos dos exames de 153 pacientes que haviam sido submetidas à histeroscopia ambulatorial após ablação do endométrio devido a sangramento uterino anormal de causa benigna, no período entre janeiro de 2006 e julho de 2011 . As pacientes foram divididas em dois grupos: $H I S T \leq 60$ (n=90), com pacientes submetidas ao exame no período de 40 a 60 dias após o procedimento, e grupo HIST>60 (n=63), das que foram examinadas entre 61 dias e 12 meses. RESULTADOS: No grupo HIST $\leq 60$, $30 \%$ das pacientes apresentavam algum grau de sinéquia; aderências grau I foram descritas em 4,4\%; grau $\mid \mathrm{em}$ 6,7\%; grau Ila em 4,4\%; grau III em 7,8\%; e 2,2\% apresentavam grau IV. No HIST>60, sinéquias foram descritas em $53,9 \%$ dos casos, 3,2\% tinham sinéquias grau $1 ; 11,1 \%$, grau $I 1 ; 7,9 \%$, grau $I$ a; $15,9 \%$, grau $I I I$; e 4,8\%, grau IV. Hematometra foi descrito em $2,2 \%$ dos casos do HIST $\leq 60$ e em $6,3 \%$ no HIST>60. CONCLUSÕES: A cavidade uterina de pacientes submetidas à histeroscopia ambulatorial até 60 dias após a ablação endometrial mostrou menor número de sinéquias quando comparada com as cavidades uterinas de pacientes que foram submetidas ao exame após 60 dias. Acompanhamento em longo prazo é necessário para avaliar plenamente o impacto da histeroscopia ambulatorial após a ablação endometrial.

\section{Abstract}

PURPOSE: To examine the aspect of the uterine cavity after hysteroscopic endometrial ablation, to determine the prevalence of synechiae after the procedure, and to analyze the importance of hysteroscopy during the postoperative period. METHODS: The results of the hysteroscopic exams of 153 patients who underwent outpatient hysteroscopy after endometrial ablation due to abnormal uterine bleeding of benign etiology during the period from January 2006 to July 2011 were retrospectively reviewed. The patients were divided into two groups: HIST $\leq 60(n=90)$ consisting of patients undergoing the exam 40-60 days after the ablation procedure, and the group HIST>60 ( $n=63$ ) consisting of patients undergoing the exam between 61 days and 12 months after the procedure. RESULTS: In the HIST $\leq 60$ group, $30 \%$ of the patients presented some degree of synechiae: synechiae grade I in $4.4 \%$ of patients, grade II in $6.7 \%$, grade $\|$ a in $4.4 \%$, grade $\|$ in $7.8 \%$, and grade IV in $2.2 \%$. In the HIST>60 group, $53.9 \%$ of all cases had synechiae, $3.2 \%$ were grade I, $11.1 \%$ grade $I$, $7.9 \%$ grade $I$ a, $15.9 \%$ grade III, and $4.8 \%$ grade IV. Hematometra was detected in $2.2 \%$ of all cases in group HIST $\leq 60$ and in $6.3 \%$ of all cases in group HIST>60. CONCLUSIONS: The uterine cavity of the patients submitted to diagnostic hysteroscopy up to 60 days after endometrial ablation showed significantly fewer synechiae compared to the uterine cavity of patients who underwent the exam after 60 days. Long-term follow-up is necessary to fully evaluate the importance of outpatient hysteroscopy after endometrial ablation regarding menstrual patterns, risk of cancer and prevalence of treatment failure.
Correspondência

Aline Rocha Guerin Hospital do Servidor Público Estadual de São Paulo Avenida Ibirapuera, 981 - Vila Clementino (EP: 04029-000 São Paulo (SP), Brasil

Recebido

$27 / 09 / 2013$

Aceito com modificacocoes

$14 / 02 / 2014$
Serviço de Ginecologia e Obstetrícia do Hospital do Servidor Público Estadual "Francisco Morato de Oliveira" - São Paulo (SP), Brasil. "Curso de Pós-Graduação, Hospital do Servidor Público Estadual "Francisco Morato de Oliveira" - São Paulo (SP), Brasil.

${ }^{2}$ Serviço de Ginecologia e Obstetrícia, Hospital do Servidor Público Estadual "Francisco Morato de Oliveira" - São Paulo (SP), Brasil. ${ }^{3}$ Setor de Endoscopia Ginecológica, Hospital do Servidor Público Estadual "Francisco Morato de Oliveira" - São Paulo (SP), Brasil. ${ }^{4}$ Seção de Ginecologia, Hospital do Servidor Público Estadual "Francisco Morato de Oliveira" - São Paulo (SP), Brasil. 


\section{Introdução}

O sangramento uterino anormal (SUA) é definido como qualquer perda sanguínea genital fora dos padrões de normalidade da menstruação e é uma importante causa de problema de saúde em mulheres ${ }^{1-3}$. A primeira opção de tratamento para o SUA é clínica, com resultados variáveis e eventualmente transitórios ${ }^{1,4,5}$. O tratamento cirúrgico é usualmente oferecido após insucesso do tratamento clínico ou quando existe contraindicação ao tratamento medicamentoso. A histerectomia é sempre eficaz, mas é associada a elevados custos econômicos e sociais, por ser um procedimento de grande porte e que pode causar complicações intra e pós-operatórias ${ }^{3,4}$. A ablação endometrial surgiu como um método cirúrgico alternativo e menos invasivo para o tratamento do sangramento uterino anormal de origem benigna ${ }^{5,6}$.

As técnicas de ablação endometrial são classificadas como histeroscópicas (primeira geração) e não histeroscópicas (segunda geração). As histeroscópicas são aquelas realizadas sob a visão direta, nas quais são utilizados instrumentos eletrocirúrgicos, como o ressectoscópico ou o laser, para destruir ou ressecar o endométrio, e que dependem da habilidade do cirurgião. As técnicas não histeroscópicas são menos complexas e requerem menor tempo para aprendizado ${ }^{1,5,7}$. As alternativas à histerectomia têm se tornado comuns nos últimos anos e provaram ser uma forma segura e efetiva de tratamento, com altas taxas de satisfação da paciente $e^{4,8-12}$.

O objetivo da ablação é destruir ou ressecar as camadas funcional e basal do endométrio e parte da camada superficial do miométrio. $\mathrm{O}$ tecido da cavidade uterina sofrerá necrose, degeneração e fibrose $e^{1,10,13,14}$.

Perfurações uterinas, sangramento pós-operatório, infecção pélvica, lesões de vísceras abdominais, embolia gasosa e sobrecarga hídrica são complicações intra e pós-operatórias precoces do procedimento, sendo a maioria evitada com boa técnica e treinamento adequado do cirurgião ${ }^{15}$.

Complicações tardias decorrem devido à proximidade das paredes anterior e posterior e à exposição do tecido miometrial ${ }^{16}$. A cavidade endometrial pode se tornar marcadamente reduzida devido à fibrose e à contratura das paredes. Se aderências e cicatrizes ocorrerem na extremidade inferior da cavidade uterina, qualquer sangramento proveniente das áreas regeneradas ou da persistência do endométrio a montante das aderências pode ficar retido e causar complicações, como hematometra central, hematometra cornual, menstruação retrógrada e síndrome da obstrução tubária pós-ablação, além de prejudicar eventual diagnóstico de carcinoma endometrial ${ }^{15-17}$. Em pacientes submetidas à ligadura tubária prévia, o sangramento retrógrado para a porção proximal da tuba pode resultar em distensão dolorosa, sendo conhecida como síndrome da ligadura tubária pós-ablação ${ }^{10}$.

A cavidade uterina pode apresentar vários graus de fibrose depois da ablação endometrial, desde curtas e finas aderências até completa obliteração ${ }^{16,17}$. Entretanto, a prevalência de aderências intrauterinas pós-ablação ainda não foi totalmente documentada ${ }^{16}$.

No seguimento pós-operatório da ablação histeroscópi$\mathrm{ca}$, alguns especialistas orientam realizar uma histeroscopia ambulatorial entre 30 a 60 dias após o procedimento cirúrgico, para realizar a lise de possíveis sinéquias que tenham se formado. Segundo esses autores, isso seria importante para manter acessível a cavidade uterina e, se no futuro a paciente necessitar de nova histeroscopia, possibilitar a avaliação $0^{1,2,18}$ da cavidade uterina.

Há poucos estudos publicados até o momento que apresentam o acompanhamento do estado da cavidade uterina após ablação endometrial histeroscópica. É importante que sejam conhecidas as consequências causadas ao endométrio após o procedimento para que, assim, seja possível avaliar se a histeroscopia ambulatorial serve como recurso para diminuir a gravidade das sinéquias.

Assim, este estudo teve por objetivo avaliar o aspecto da cavidade uterina após a ablação endometrial histeroscópica, a prevalência de sinéquias após o procedimento e, com isso, avaliar a importância da histeroscopia realizada no pós-operatório dessas pacientes.

\section{Métodos}

Este é um estudo retrospectivo, no qual foram analisados os registros médicos de todas as pacientes submetidas consecutivamente à histeroscopia ambulatorial no Setor de Endoscopia Ginecológica do Serviço de Ginecologia e Obstetrícia do Hospital do Servidor Público Estadual de São Paulo, no período entre janeiro de 2006 e julho de 2011. Este estudo retrospectivo, baseado em prontuários, foi aprovado pelo Comitê de Ética em Pesquisa do Hospital.

Foram incluídas no estudo pacientes com diagnóstico de sangramento uterino anormal, sem sucesso com o tratamento clínico, com histeroscopia ambulatorial prévia, que foram submetidas à ablação endometrial histeroscópica.

Todas as pacientes incluídas no estudo foram tratadas, previamente ao procedimento de ablação, com gosserrelina, na dose de 3,6 mg por mês durante dois ou três meses, ou 10,8 mg em uma única aplicação. O análogo do hormônio liberador das gonadotrofias $(\mathrm{GnRH})$ foi utilizado para diminuir a espessura do endométrio, facilitando o procedimento cirúrgico.

O procedimento foi realizado em centro cirúrgico com as pacientes sob analgesia de bloqueio. Todos os 
procedimentos foram realizados pela equipe de endoscopia ginecológica do mesmo hospital.

A técnica de ablação histeroscópica do endométrio utilizada no serviço foi a ressecção com corrente elétrica monopolar, usando-se o ressectoscópio de 26 Frenchs, ótica de $4 \mathrm{~mm}$ e 30 graus. Após a dilatação cervical com velas de Hegar até o número 9,5, o ressectoscópio foi introduzido na cavidade uterina. $\mathrm{O}$ procedimento começou com a ressecção com alça em "U", modo corte e potência de $90 \mathrm{~W}$, tendo como ponto de partida as paredes posterior, laterais e, por último, anterior, sendo preservada uma região de aproximadamente $1 \mathrm{~cm}$ de endométrio a partir do orifício interno do colo uterino. Em seguida, foi realizada a destruição do tecido endometrial próximo aos óstios tubários e do fundo uterino com a alça rollerball, modo coagulação, com potência de $90 \mathrm{~W}$. Por último, com a alça rollerball, foi feita a hemostasia. Os histeroscópios usados foram da marca Wolff e Storz. O meio de distensão utilizado foi a glicina a $1,5 \%$, com pressão e fluxo controlados pelo histerodistensor. Todo o material ressecado foi enviado para avaliação histológica no Serviço de Anatomia Patológica do hospital.

No seguimento pós-operatório, as pacientes foram orientadas a retornar no período de 40 a 60 dias após o procedimento cirúrgico para uma histeroscopia ambulatorial, conforme rotina do Serviço. Todas as histeroscopias diagnósticas foram realizadas pela mesma equipe.

As histeroscopias foram realizadas pela técnica da vaginoscopia descrita por Bettocchi e Selvaggi ${ }^{19}$, que consiste em uma abordagem sem necessidade de toque vaginal, sem uso de espéculo e sem pinçamento cervical. Foi utilizada ótica de $2,9 \mathrm{~mm}$ com ângulo de 30 graus, com camisa interna de influxo, diâmetro total de $4 \mathrm{~mm}$ e extremidade ovalada (Histeroscópio de Bettocchi, Karl Storz, Alemanha). Para a distensão vaginal e uterina, foi utilizado soro fisiológico a $0,9 \%$, em temperatura ambiente, com pressão determinada por gravidade e enchimento de manguito de pressão ao redor de frasco flexível, com pressão suficiente para adequada visibilização do canal cervical e da cavidade uterina. A imagem foi transmitida para um monitor e a fonte de luz utilizada foi a de xênon de $300 \mathrm{~W}$. A técnica consistiu na colocação da ponta da ótica no vestíbulo vaginal, abertura da torneira para liberação da entrada do líquido e, com isso, distensão da vagina, permitindo visibilização do colo e penetração no orifício externo, não sendo necessário aproximar ou ocluir os lábios vaginais ${ }^{20}$.

À revisão dos prontuários, 153 pacientes puderam ser incluídas no estudo e foram divididas em dois grupos: HIST $\leq 60$, de pacientes que se submeteram ao exame no período orientado pela equipe $(n=90)$, e HIST $>60$, de pacientes que não fizeram o exame no período solicitado, retornando para a histeroscopia somente entre 61 dias e 12 meses após o procedimento cirúrgico $(\mathrm{n}=63)$. Pacientes que retornavam após um ano eram excluídas do estudo.

A média das idades das pacientes do grupo HIST $\leq 60$ no momento da ablação endometrial foi de 46,4 anos (36-56) e a paridade média foi de três filhos (0-8). O volume uterino médio foi de 125, 2 cc (37-250). No grupo HIST > 60, a média de idade foi de 45,2 anos (35-63), a de paridade foi de dois filhos (0-5) e a média do volume uterino no momento da ablação de 131 cc (46-243). Todas as mulheres se encontravam na menacme, com sangramento uterino anormal não responsivo ao tratamento clínico.

Os laudos desses exames foram analisados a partir da observação dos seguintes aspectos: a forma da cavidade uterina, a visibilização dos óstios tubários, o aspecto do endométrio e a presença de sinéquias. As sinéquias foram classificadas de acordo com o proposto por Wamsteker e De Block ${ }^{21}$, da European Society of Gynaecological Endoscopy (ESGE) (Quadro 1).

Foram questionados, durante a histeroscopia, os padrões menstruais das mulheres em acompanhamento. A intervenção era considerada bem-sucedida quando a paciente permanecia em amenorreia, hipomenorreia ou eumenorreia.

Também foram coletados dados sobre os achados de doenças endometriais durante a histeroscopia, como miomas, pólipos, hiperplasias ou cicatriz hipertrófica de cesárea.

Foi realizado o teste da razão de verossimilhanças para verificar a existência de associação entre os grupos de histeroscopia e os aspectos da cavidade uterina. Para comparar os graus de sinéquias entre os grupos de histeroscopia, foi utilizado o teste Mann-Whitney. As análises estatísticas foram realizadas com uso do software Statistical Package for the Social Sciences (SPSS) versão 20.0 e os testes foram realizados com nível de significância de $5 \%$.

Quadro 1. Classificação de sinéquias proposta pela European Society of Gynaecological Endoscopy ${ }^{21}$

\begin{tabular}{|c|c|}
\hline Grau & Extensão das aderências intrauterinas \\
\hline 1 & $\begin{array}{l}\text { Aderências finas, facilmente rompidas pela bainha do histeroscópio. } \\
\text { Áreas cornuais normais. }\end{array}$ \\
\hline$\|$ & $\begin{array}{l}\text { Aderência fina, única, conectando partes separadas da cavidade uterina. A visibilização de } \\
\text { ambos os óstios das tubas é possível. Não pode ser rompida com a bainha do histeroscópico. }\end{array}$ \\
\hline$\|_{a}$ & $\begin{array}{l}\text { Oclusão somente na região do orifício interno do colo uterino. A região superior da cavidade } \\
\text { uterina é normal. }\end{array}$ \\
\hline III & $\begin{array}{l}\text { Múltiplas aderências firmes, ligando partes separadas da cavidade uterina. Obliteração } \\
\text { unilateral da região dos óstios tubários. }\end{array}$ \\
\hline IV & $\begin{array}{l}\text { Aderências densas extensas com parcial oclusão da cavidade. Ambas as áreas dos óstios } \\
\text { tubários ocluídos (parcialmente). }\end{array}$ \\
\hline $\mathrm{Va}$ & $\begin{array}{l}\text { Extenso dano endometrial e fibrose em combinação com aderências de grau I ou II } \\
\text { com amenorreia ou acentuada hipomenorreia. }\end{array}$ \\
\hline $\mathrm{Vb}$ & $\begin{array}{l}\text { Extenso dano endometrial e fibrose em combinação com aderências de grau III e IV } \\
\text { com amenorreia. }\end{array}$ \\
\hline
\end{tabular}


Resultados

Conforme descrito, o grupo HIST $\leq 60$ foi composto por 90 pacientes que foram submetidas à histeroscopia ambulatorial em até 60 dias de pós-operatório. Em 42 casos, o endométrio foi descrito como cicatricial, apresentando áreas de necrose, granulomas e com presença de fibrina. Em 20 casos, o endométrio estava atrófico e em 19, tinha áreas de atividade proliferativa. Também foram descritos três casos de endométrio misto e dois casos de endométrio proliferativo com lesões arroxeadas sugestivas de adenomiose. Pólipos endometriais foram diagnosticados em seis casos, variando de 0,5 a $3,5 \mathrm{~cm}$, e miomas uterinos foram visibilizados em cinco casos, sendo três miomas classificados como tipo I e dois como tipo $\mathrm{II}^{22}$. Cicatriz hipertrófica de cesárea foi descrita em seis pacientes.

Quanto à forma da cavidade uterina (Tabela 1), 59 pacientes $(65,6 \%)$ tinham cavidade irregular, $16(17,8 \%)$ cavidade regular, $11(12,2 \%)$ cavidade de aspecto tubular e $4(4,4 \%)$ estavam com obstrução do orifício cervical interno.

Do total, 30\% das pacientes apresentavam algum grau de sinéquia (Tabela 2). Sinéquias grau I foram encontradas em quatro pacientes $(4,4 \%)$, sinéquias grau II em seis $(6,7 \%)$ e aderências de grau IIa em quatro $(4,4 \%)$ casos. Sinéquias grau III foram descritas em sete $(7,8 \%)$

Tabela 1. Descrição do aspecto das cavidades uterinas segundo grupos e resultado do teste de associação

\begin{tabular}{|c|c|c|c|c|c|}
\hline \multirow[t]{2}{*}{ Aspecto da cavidade } & \multicolumn{2}{|c|}{$\begin{array}{l}\text { Histeroscopia } \\
\text { até } 60 \text { dias }\end{array}$} & \multicolumn{2}{|c|}{$\begin{array}{c}\text { Histeroscopia } \\
\text { mais de } 60 \text { dias }\end{array}$} & \multirow[t]{2}{*}{ Valor p* } \\
\hline & n & $\%$ & n & $\%$ & \\
\hline Cavidade tubular & 11 & 12,2 & 9 & 14,3 & 0,2 \\
\hline Cavidade regular & 16 & 17,8 & 14 & 22,2 & \\
\hline Cavidade irregular & 59 & 65,6 & 33 & 52,4 & \\
\hline $\begin{array}{l}\text { Obstrução do orifício } \\
\text { cervical interno }\end{array}$ & 4 & 4,4 & 7 & 11,1 & \\
\hline Total & 90 & 100 & 63 & 100 & \\
\hline
\end{tabular}

*Resultado do teste da razão de verossimilhanças.

Tabela 2. Descrição dos graus das sinéquias segundo grupos e resultado do teste comparativo

\begin{tabular}{|c|c|c|c|c|c|}
\hline \multirow[t]{2}{*}{ Sinéquias } & \multicolumn{2}{|c|}{$\begin{array}{l}\text { Histeroscopia } \\
\text { até } 60 \text { dias }\end{array}$} & \multicolumn{2}{|c|}{$\begin{array}{l}\text { Histeroscopia } \\
\text { mais de } 60 \text { dias }\end{array}$} & \multirow[t]{2}{*}{ Valor $\mathrm{p}^{*}$} \\
\hline & n & $\%$ & n & $\%$ & \\
\hline Sem sinéquias & 67 & 74,5 & 36 & 57,1 & 0,004 \\
\hline Grau I & 4 & 4,4 & 2 & 3,2 & \\
\hline Grau II & 6 & 6,7 & 7 & 11,1 & \\
\hline Grau lla & 4 & 4,4 & 5 & 7,9 & \\
\hline Grau III & 7 & 7,8 & 10 & 15,9 & \\
\hline Grau IV & 2 & 2,2 & 3 & 4,8 & \\
\hline Total & 90 & 100 & 63 & 100 & \\
\hline
\end{tabular}

*Resultado do Teste de Mann-Whitney. pacientes e em dois casos $(2,2 \%)$ as aderências foram de grau IV. O achado de hematometra foi descrito em duas pacientes $(2,2 \%)$.

Entre as pacientes deste grupo, 2 (2,2\%) não apresentaram melhora do sangramento uterino anormal, 76 $(84,5 \%)$ tinham amenorreia e $12(13,3 \%)$ estavam em hipomenorreia.

No grupo HIST >60, com 63 pacientes, em 33 casos $(52,3 \%)$, a cavidade uterina tinha aspecto irregular e em 14 (22,2\%), regular (Tabela 1). Cicatriz uterina grave, deixando a cavidade de formato tubular, foi vista em 9 casos $(14,2 \%)$. Obliteração do orifício interno que dificultou a progressão do histeroscópio foi registrada em 7 pacientes $(11,1 \%)$. Cavidades apresentando endométrio cicatricial, com fragmentos de endométrio e granulomas, foram descritas em quatro casos. Endométrio misto foi descrito em 7 casos e atrófico em 15. Em 30 casos, o endométrio tinha aspecto de atividade proliferativa. Nestes, seis possuíam miomas, sendo dois miomas tipo 0, um tipo I e três tipo II. Pólipos foram encontrados em 13 pacientes. Cicatriz hipertrófica de cesárea foi observada em cinco casos.

Sinéquias uterinas foram descritas em $53,9 \%$ dessas pacientes (Tabela 2). Duas pacientes $(3,2 \%)$ possuíam sinéquias grau I. Aderências grau II foram encontradas em 7 casos $(11,1 \%)$. Cinco mulheres $(7,9 \%)$ tinham aderências grau IIa. Múltiplas aderências firmes, com obliteração de um dos óstios, classificadas em grau III, foram encontradas em 10 casos $(15,9 \%)$. Aderências grau IV foram vistas em três casos $(4,8 \%)$. A presença de hematometra neste grupo foi descrita em quatro casos $(6,3 \%)$.

Dentre as pacientes deste grupo, 34 (54\%) estavam em amenorreia, $19(30,1 \%)$ em hipomenorreia ou eumenorreia, e $10(15,9 \%)$ não apresentaram melhora do sangramento uterino anormal.

Em relação ao aspecto da cavidade uterina, não foi verificada associação significativa com os grupos $(\mathrm{p}=0,2)$ (Tabela 1). Já os graus de sinéquias foram significativamente mais graves nas pacientes com histeroscopia com mais de 60 dias $(\mathrm{p}=0,004)$ (Tabela 2).

\section{Discussão}

A ablação endometrial é um dos tratamentos seguros e efetivos estabelecidos para o sangramento uterino anormal $^{23}$. Como observado no presente estudo, a destruição do endométrio e a consequente reação inflamatória aguda podem resultar na formação de aderências intrauterinas que podem obstruir qualquer eliminação de sangue residual do endométrio. Entretanto, poucos estudos avaliaram a aparência da cavidade uterina depois da ablação endometrial histeroscópica. 
Istre et al. ${ }^{17}$ realizaram histeroscopia de controle em 58 mulheres 1 ano após a ressecção endometrial e comentam, sem, no entanto, descrever a frequência, que a cavidade uterina geralmente apareceu como um tubo estreito. No presente estudo, a frequência encontrada de cavidade de aspecto tubular foi de 12,2\% no grupo HIST $\leq 60$ e 14,2\% no grupo HIST $>60$. Este é um resultado esperado da fibrose e da oclusão da parte periférica da cavidade. Os mesmos autores também descrevem que encontraram, após 12 meses da ressecção endometrial, aderências focais em $40 \%$ dos casos, porém nenhuma mulher havia desenvolvido obliteração total da cavidade. Fato semelhante foi observado neste estudo no grupo HIST > 60, em que foram encontradas sinéquias em $42,8 \%$ dos casos. Já no grupo HIST $\leq 60$, a quantidade de sinéquias foi menor. Essas diferenças podem ser explicadas por um tempo de cicatrização maior no grupo HIST $>60$, sem ocorrer a lise das sinéquias pela passagem do histeroscópio em um pós-operatório mais precoce.

As taxas de ocorrência de hematometra central são semelhantes entre os vários estudos com seguimento de pacientes após ablação endometrial. Assi, foi verificado $1 \%$ em uma pequena casuística de $24 \operatorname{casos}^{24} ; 1 \%$ após seguimento em longo prazo (24 a 36 meses $)^{25}$ e 2,4\% em uma série de $114 \operatorname{casos}^{11}$. Na presente casuística, a prevalência de hematometra foi de 2,2\% no grupo HIST $\leq 60$ e 6,3\% no grupo HIST > 60, demonstrando neste último taxa bem mais elevada que a encontrada em outros estudos ${ }^{24-26}$. Essa diferença questiona se a realização da histeroscopia ambulatorial em um pós-operatório precoce não destruiria as sinéquias em formação e diminuiria a prevalência de hematometra neste grupo. Hematometra central ocorre geralmente quando o canal cervical foi lesado durante a ablação endometrial ${ }^{26}$.

Onoglu et al..$^{27}$ publicaram estudo comparativo mostrando áreas focais de endométrio regenerado no fundo e óstios em grupos que se submeteram à ablação endometrial, tanto com rollerball como com ressectoscópio.

Estudos com ressonância magnética mostraram que o tecido endometrial pode estar presente em até 95\% das pacientes submetidas à ablação endometrial $^{28}$. Empregando-se biópsias endometriais, pode ser comprovada a presença de endométrio regenerado em $25 \%$ das pacientes com amenorreia, 3 meses depois da ressecção endometrial ${ }^{29}$. Os achados deste estudo mostraram que o ressurgimento do endométrio esteve presente em $26,6 \%$ das pacientes submetidas à histeroscopia ambulatorial em 40 a 60 dias da cirurgia e em $58,7 \%$ naquelas que fizeram com mais de 60 dias. O ressurgimento do endométrio é um desenvolvimento esperado em muitas pacientes e não está necessariamente associado com o SUA após a cirurgia ${ }^{27-30}$. O ressurgimento do endométrio poderia ser considerado uma falha do tratamento de ablação, porém pode ocorrer em mulheres clinicamente amenorreicas, persistindo o risco de neoplasia endometrial ${ }^{28,31}$.

Em vários estudos publicados, a taxa de sucesso da ablação endometrial (ou seja, a interrupção ou diminuição do sangramento) está em torno de 80-90\%, com risco de $15-35 \%$ de as pacientes precisarem ser submetidas a nova ablação endometrial ou histerectomia nos primeiros 5 anos $^{5,32}$. Com maior tempo de seguimento, a taxa de sucesso da ablação endometrial tem variado de $67-92 \% \%^{9,11,33}$. Este estudo encontrou taxa de sucesso maior do que a observada em outros estudos: de 97,8\% para pacientes avaliadas até 2 meses após o procedimento e de $84,2 \%$ para pacientes avaliadas até 1 ano após o procedimento. No entanto, este estudo foi feito em curto período após a ablação, sendo possíveis mudanças desses valores em médio e longo prazo.

No presente estudo, foi verificado que o grau das sinéquias intrauterinas torna-se progressivamente mais grave com o tempo. Apesar de a formação das sinéquias ser resposta esperada após a ablação endometrial, a gravidade delas pode dificultar uma futura avaliação da cavidade uterina. A histeroscopia ambulatorial precoce pós-ablação endometrial parece ser útil para diagnóstico e tratamento das sinéquias intrauterinas que ocorrem após a ablação.

Como este trabalho não foi um estudo longitudinal, com estes dados, isoladamente, não é possível inferir a necessidade e a importância da histeroscopia ambulatorial após a ablação endometrial. Acompanhamento em longo prazo é necessário para avaliar plenamente o valor da histeroscopia ambulatorial após a ablação endometrial em relação aos padrões menstruais, risco de câncer e incidência de falha do tratamento.

\section{Referências}

1. Lopes MC, Panisset KSP, Marques WS. Ablação endometrial. In: Crispi CP, Oliveira FM, Damian Junior JC, Oliveira MA, Ribeiro PA. Tratado de endoscopia ginecológica: cirurgia minimamente invasiva. 3a ed. Rio de Janeiro: Revinter; 2012. p. 696-710.

2. Lethaby A, Hickey M, Garry R. Endometrial destruction techniques for heavy menstrual bleeding. Cochrane Database Syst Rev. 2005;(4):CD001501.
3. Fergusson R, Lethaby A, Shepperd S, Farquhar C. Endometrial resection and ablation versus hysterectomy for heavy menstrual bleeding. Cochrane Database Syst Rev. 2013;11:CD000329.

4. David SD, Depes DB, Ramos JFD, Lopes RGC, Lippi UG, Baracat $F F$, et al. Ressecção histeroscópica do endométrio: eficácia e fatores relacionados ao insucesso. Einstein (São Paulo). $2008 ; 6(2): 120-4$. 
5. Garry R. Evidence and techniques in endometrial ablation: consensus. Gynaecol Endosc. 2002; 11 (1):5-17.

6. Bøe Engelsen I, Woie K, Hordnes K. Transcervical endometrial resection: long-term results of 390 procedures. Acta Obstet Gynecol Scand. 2006;85(1):82-7.

7. Glasser MH. Practical tips for office hysteroscopy and secondgeneration "global" endometrial ablation. J Minim Invasive Gynecol. 2009; 16(4):384-99.

8. Cooper JM, Anderson TL, Fortin CA, Jack SA, Plentl MB. Microwave endometrial ablation vs. rollerball electroablation for menorrhagia: a multicenter randomized trial. J Am Assoc Gynecol Laparosc. 2004; 11 (3):394-403.

9. Comino R, Torrejón R. Hysterectomy after endometrial ablationresection. J Am Assoc Gynecol Laparosc. 2004; 11 (4):495-9.

10. Lopes RG. Ablação do endométrio. In: Lopes RG, editor. O endométrio. São Paulo: Atheneu; 2011 . p. 305-21.

11. Takahashi WH, Lopes RG, Depes DB, Castello Branco HK. [Results of hysteroscopic endometrial ablation after five-year follow-up]. Rev Bras Ginecol Obstet. 2012;34(2):80-5. Portuguese.

12. Riley KA, Davies MF, Harkins GJ. Characteristics of patients undergoing hysterectomy for failed endometrial ablation. JSLS. 2013;17(4):503-7.

13. Boujida VH, Philipsen T, Pelle J, Joergensen JC. Five-year follow-up of endometrial ablation: endometrial coagulation versus endometrial resection. Obstet Gynecol. 2002;99(6):988-92.

14. Luo X, Lim CE, Li L, Wong WS. Hysteroscopic appearance of endometrial cavity after microwave endometrial ablation. J Minim Invasive Gynecol. 2010;17(1):30-6.

15. Sharp HT. Endometrial ablation: what counts? Obstet Gynecol. 2010;1 16(4):808-9.

16. Leung PL, Tam WH, Yuen PM. Hysteroscopic appearance of the endometrial cavity following thermal balloon endometrial ablation. Fertil Steril. 2003;79(5):1226-8.

17. Istre O, Skajaa K, Holm-Nielsen P, Forman A. The second-look appearance of the uterine cavity after resection of the endometrium. Gynaecol Endosc. 1993;2(3):159-63.

18. Donnez J, Polet R, Rabinovitz R, Ak M, Squifflet J, Nisolle M. Endometrial laser intrauterine thermotherapy: the first series of 100 patients observed for 1 year. Fertil Steril. 2000;74(4):791-6.

19. Bettocchi S, Selvaggi L. A vaginoscopic approach to reduce the pain of office hysteroscopy. J Am Assoc Gynecol Laparosc. 1997;4(2):255-8.
20. Bettocchi S, Nappi L, Ceci O, Selvaggi L. What does 'diagnostic hysteroscopy' mean today? The role of the new techniques. Curr Opin Obstet Gynecol. 2003;15(4):303-8.

21. Wamsteker K, De Block S. Diagnostic hysteroscopy: technique and documentation. In: Sutton C, Diamond MP, editors. Endoscopic surgery for gynaecologists. London: WB Saunders; 1998. p. $511-24$.

22. Polena V, Mergui JL, Perrot N, Poncelet C, Barranger E, Uzan S. Long-term results of hysteroscopic myomectomy in 235 patients. Eur J Obstet Gynecol Reprod Biol. 2007;130(2):232-7.

23. Litta P, Merlin F, Pozzan C, Nardelli GB, Capobianco G, Dessole S, et al. Transcervical endometrial resection in women with menorrhagia: long-term follow-up. Eur J Obstet Gynecol Reprod Biol. 2006; 125(1):99-102.

24. Hill DJ. Hematometra - a complication of endometrial ablation/ resection. J Am Assoc Gynecol Laparosc. 1994; 1 (4, Part 2):S1 4.

25. Martyn P, Allan B. Long-term follow-up of endometrial ablation. J Am Assoc Gynecol Laparosc. 1998;5(2):115-8.

26. El-Nashar SA, Hopkins MR, Creedon DJ, St Sauver JL, Weaver AL, $M c G r e e M E$, et al. Prediction of treatment outcomes after global endometrial ablation. Obstet Gynecol. 2009;1 13(1):97-106.

27. Onoglu A, Taskin O, Inal M, Sadik S, Simsek M, Akar M, et al. Comparison of the long-term histopathologic and morphologic changes after endometrial rollerball ablation and resection: a prospective randomized trial. J Minim Invasive Gynecol. $2007 ; 14(1): 39-42$.

28. Turnbull LW, Jumaa A, Bowsley SJ, Dhawan S, Horsman A, Killick SR. Magnetic resonance imaging of the uterus after endometrial resection. Br J Obstet Gynaecol. 1997;104(8):934-8.

29. Magos AL, Baumann R, Lockwood GM, Turnbull AC. Experience with the first 250 endometrial resections of menorrhagia. Lancet. 1991;337(8749): 1074-8.

30. Pérez-Medina T, Haya J, Frutos LS, Arenas JB. Factors influencing long-term outcome of loop endometrial resection. J Am Assoc Gynecol Laparosc. 2002;9(3):272-6.

31. Sagiv R, Ben-Shem E, Condrea A, Glezerman M, Golan A. Endometrial carcinoma after endometrial resection for dysfunctional uterine bleeding. Obstet Gynecol. 2005; $106(5$ Pt 2):1 174-6.

32. Dutton C, Ackerson L, Phelps-Sandall B. Outcomes after rollerball endometrial ablation for menorrhagia. Obstet Gynecol. $2001 ; 98(1): 35-9$.

33. Fürst SN, Philipsen T, Joergensen JC. Ten-year follow-up of endometrial ablation. Acta Obstet Gynecol Scand. 2007;86(3):334-8. 